\title{
Preface to the GPSD 2012 special issue of Metrika
}

\author{
Nicole Bäuerle • Herold Dehling • Achim Klenke • \\ Götz Kersting • Jens-Peter Kreiss
}

Received: 16 September 2013 / Published online: 3 December 2013

(C) Springer-Verlag Berlin Heidelberg 2013

This issue of Metrika is devoted to the topics of the invited lectures of the 10th German Probability and Statistics Days (GPSD 2012) that was held in March 2012 at the University of Mainz. The conference was organized by the Probability and Statistics section of the German Mathematical Society (DMV-Fachgruppe Stochastik), in cooperation with the Institute of Mathematics of the Johannes Gutenberg University Mainz. The German Probability and Statistics Days are held biannually, with previous meetings at Marburg 1993, Freiberg 1996, München 1998, Hamburg 2000, Magdeburg 2002, Karlsruhe 2004, Frankfurt 2006, Aachen 2008 and Leipzig 2010. The next meeting in this series will be held in 2014 at the University of Ulm.

The DMV-Fachgruppe Stochastik was founded in 1993 as a section of the German Mathematical Society DMV, with the aim to promote the development of Probability Theory and Mathematical Statistics in Germany. Currently, the Fachgruppe Stochastik has more than 400 members, representing most of the probabilists and statisticians working at Mathematics departments in Germany. In addition to the GPSD series of conferences, the Fachgruppe Stochastik organizes annual workshops for Ph.D. stu-

\footnotetext{
N. Bäuerle

Institut für Stochastik, Karlsruher Institut fur Technologie, Karlsruhe, Germany

H. Dehling $(\bowtie)$

Fakultät für Mathematik, Ruhr-Universität Bochum, Universitätsstraße 150, 44780 Bochum, Germany e-mail: herold.dehling@rub.de
}

\author{
A. Klenke \\ Institut für Mathematik, Johannes Gutenberg-Universität Mainz, Mainz, Germany \\ G. Kersting \\ Institut für Mathematik, Goethe-Universität Frankfurt am Main, Frankfurt, Germany \\ J.-P. Kreiss \\ Institut für Mathematische Stochastik, Technische Universität Braunschweig, Braunschweig, Germany
}


dents and awards prizes for outstanding recent Ph.D. theses. The Fachgruppe maintains a moderated e-mail server, which is used to disseminate information of interest to probabilists and statisticians in Germany and abroad, with currently more than 1000 subscribers. In 2013, the Fachgruppe co-organized its first conference outside of Germany, namely the First German-Polish Joint Conference on Probability and Mathematical Statistics, which was held in June 2013 in Torun (PL).

Within 20 years, the German Probability and Statistics Days have grown from a small local conference with mostly German participants to its present format with more than 500 participants from all over Europe. In this way, the GPSD conferences are now among the major probability and statistics conferences in Europe. The GPSD 2012 had almost 400 scientific talks. In addition to four plenary talks, there were 14 sections, devoted to the topics 1. Stochastic Analysis, 2. Limit Theorems, Large Deviations and Extremes, 3. Stochastic Geometry, Spatial Statistics and Image Analysis, 4. Random Discrete Structures and Analysis of Algorithms, 5. Stochastic Processes, 6. Stochastics in the Sciences, 7. Stochastic Models in Engineering, 8. Statistics and Decision Theory, 9. Nonparametric Statistics and Curve Estimation, 10. Statistics of Processes and Time Series, 11. Applied Statistics and Data Analysis, 12. Stochastic Optimization and Operations Research, 13. Finance, Insurance, Risk: Modeling, and 14. Finance, Insurance, Risk: Statistics. In addition, there was a section devoted to the teaching of probability and statistics in schools, promoting the interaction of university researchers and high school teachers. For more details about the programme, see the conference website http://gpsd2012.uni-mainz.de/.

We are grateful to the Metrika editors Holger Dette and Udo Kamps for inviting us to publish selected papers from the GPSD 2012 conference in a special issue of Metrika. The members of the board of the Fachgruppe Stochastik served as associate editors. This special issue contains seven papers from invited speakers at GPSD 2012. The invited speakers who contributed to this issue were Valentine Genon-Catalot (Paris), Remco van der Hofstad (Eindhoven), Gregory Lawler (Chicago), Mikhail Nikulin (Bordeaux), Francesco Russo (Paris), Richard Stockbridge (Milwaukee), and Murad Taqqu (Boston). The topics of the papers range from Stochastic Analysis to Applied Statistics.

In their paper Note on the existence and modulus of continuity of the $S L E_{8}$ curve, Marcelo Alvisio and Gregory Lawler review a particular approach to construct the Schramm-Loewner Evolution $S L E_{8}$ with index $\kappa=8$ using a reverse Loewner equation and a specific Girsanov transform. They show how this construction could be used to attack the problem of bounding the modulus of continuity of the $S L E_{8}$ curve if some crucial problems could be resolved. However, they also show that these problems are substantial and hence the solution has to wait.

For bond percolation on an infinite transitive graph, the validity of the so-called triangle condition ensures similarity to percolation on a regular tree. More precisely, many of the critical exponents coincide. In their paper Unlacing hypercube percolation: a survey, Remco van der Hofstad and Asaf Nachmias give a new proof for the triangle condition for percolation on hypercubes that works without the celebrated method of lace expansions. In their paper, they also give a review on results for hypercube percolation. 
The paper The covariation for Banach space valued processes and applications of Cristina Di Girolami, Giorgio Fabbri and Francesco Russo reviews recent developments in the stochastic calculus of Banach space valued processes. One interesting example is the window process of a real-valued process, made up by sections of its paths. Covariation and quadratic variation are introduced via regularization, which differs somewhat from the classical approach. Generalizations of the concepts of Ito processes and semimartingales are discussed. Applications concern the robustness of the well-known Clark-Okone formula under change of the underlying process and the probabilistic representation of stochastic partial differential equations.

The paper Hermite ranks and U-statistics by Céline Lévy-Leduc and Murad Taqqu investigates the asymptotic behavior of bivariate U-statistics in the case of long-range dependent data. The authors propose an approach based on an $L_{2}$-expansion of the kernel function into a series of bivariate Hermite polynomials. Since the observations are dependent, the pair distributions differ, and thus the Hermite expansion is not uniquely defined. The authors propose three different Hermite expansions, and define the associated Hermite coefficients and Hermite ranks. They compare these ranks, provide formulas for relating the Hermite coefficients in the different expansions, and give conditions under which the remainder terms are asymptotically negligible. The authors apply their techniques to several important kernels and determine the asymptotic distribution of the corresponding U-statistics.

The paper Discussion of Dynamic Programming and Linear Programming Approaches to Stochastic Control and Optimal Stopping in Continuous Time by Richard Stockbridge seeks to highlight two approaches to the solution of stochastic control and optimal stopping problems in continuous time. The first one is the well-known Dynamic Programming (DP) approach and the second one is the Linear Programming (LP) approach. Both approaches make use of the Dynkin formula and transform the problem into a deterministic optimization problem but in essentially different ways. Whereas the DP approach leads to a partial/ordinary differential equation or variational or quasivariational inequality depending on the type of problem, the LP approach leads to a linear program over a space of feasible measures. The paper explains these methods in the framework of multidimensional diffusions for problems with a finite time horizon, problems with control until first exit of a region and problems with discounted cost and singular control. Optimal stopping problems are considered in greater detail. A lot of illustrating examples are also given and the exposition is mainly non technical.

The paper Nonparametric density estimation in compound Poisson processes using convolution power estimators of Fabienne Comte, Céline Duval and Valentine GenonCatalot considers a compound Poisson process which can be observed on an equidistant discrete time grid. The authors suggest and investigate according to $L_{2}$ risk a nonparametric estimator of the jump size density, which is based on a truncated series approximation of a specific operator which is applied to the density of the observed increments (conditionally that the increment is not zero), which in turn is a series of weighted convolutions of the underlying density. Advantages of the proposed estimator are that it can easily be implemented and that it performs rather well, as is shown in a simulation study. The paper nicely extends the existing literature on jump size density estimation for discretely observed Poisson processes. 
Goodness of fit tests for parametric hypotheses of the transition intensities of homogeneous as well as nonhomogeneous Markov processes in discrete time and finite state spaces are the focus of interest in the paper Goodness-of-fit tests for parametric nonhomogeneous Markov processes of Vilijandas Bagdonavičius and Mikhail Nikulin. The proposed test statistic is based on the difference of a nonparametric and a parametric maximum likelihood estimator of the expected number of transitions from one state to another. Under suitable regularity conditions, which allow for an asymptotic linear expansion of the maximum likelihood estimator, the authors establish for a properly chosen quadratic form an asymptotic chi-square distribution under the null. The authors apply their results to a variety of examples and obtain relevant statistical tests for several well-known models. 